\title{
Aplicación del diseño D-Optimal en la modelación de la adsorción de COVs-Cl sobre zeolitas naturales chilenas modificadas
}

\author{
Application of D-optimal design for modelling the adsorption \\ of Cl-VOCs onto modified natural zeolites
}

Andrés L. Riquelme ${ }^{1} \quad$ Héctor Valdés ${ }^{1 *}$

Recibido 16 de mayo de 2014, aceptado 21 de marzo de 2016

Received: May 16, 2014 Accepted: March 21, 2016

\begin{abstract}
RESUMEN
En este trabajo se investigaron las variables de diseño y los parámetros operacionales en el proceso de adsorción de compuestos orgánicos volátiles clorados $(\mathrm{COVs}-\mathrm{Cl})$ utilizando zeolita natural modificada con metales de transición (ZMt). Se establecieron los niveles de las variables de diseño: temperatura de desgasificación $\left[350^{\circ} \mathrm{C}, 550^{\circ} \mathrm{C}\right]$, tipo de metal de transición $[\mathrm{Fe}, \mathrm{Mn}, \mathrm{Ag}, \mathrm{Co}, \mathrm{Cu}, \mathrm{Ni}$, concentración del metal depositado [0,05; 0,1 M], método de deposición del metal [impregnación, intercambio iónico], posttratamiento del metal depositado [desgasificación con Ar, calcinación con $\mathrm{O}_{2}$, reducción con $\mathrm{H}_{2}$ ], y como parámetros operacionales: tipo de $\mathrm{COVs}-\mathrm{Cl}$ [percloroetileno, clorobenceno], concentración de $\mathrm{COVs}-\mathrm{Cl}$ $\left[1,5 ; 9,5 \mu \mathrm{mol} \mathrm{dm}{ }^{-3}\right]$, temperatura de adsorción $\left[20 ; 100^{\circ} \mathrm{C}\right]$, flujo volumétrico de COVs-Cl $\left[50 ; 100 \mathrm{~cm}^{3}\right.$ $\left.\mathrm{min}^{-1}\right]$, masa de ZMt [0,15; 0,3 g]. Se utilizó el diseño de experimento D-Optimal, con el que el número de experimentos a realizar fue reducido de 2.048 a 139. Los experimentos de adsorción se llevaron a cabo en un adsorbedor de lecho fijo, siguiendo la concentración del COVs-Cl por cromatografía de gases. Los resultados experimentales se ajustaron a un modelo de regresión lineal de segundo orden. El análisis de varianza (ANOVA) demostró que el modelo ajustado es altamente significativo y con una satisfactoria bondad de ajuste. Los resultados del ANOVA también indicaron que entre las variables significativas, las zeolitas intercambiadas con $\mathrm{Fe}$ y $\mathrm{Cu}$ tienen un efecto positivo en la capacidad de adsorción de la ZMt. La concentración de $\mathrm{COVs-Cl}$ y temperatura de adsorción muestran la mayor influencia en la eliminación de COVs-Cl entre las variables de operación estudiadas.
\end{abstract}

Palabras clave: Adsorción, ANOVA, COVs-Cl, D-Optimal, modelo de segundo orden, zeolita modificada.

\section{ABSTRACT}

In this work, design variables and operating conditions in the adsorption of chlorinated volatile organic compounds (Cl-VOCs) using transition metal-modified natural zeolites (ZMt) are investigated. The levels of design variables are established: out-gassing temperature $\left[350{ }^{\circ} \mathrm{C}, 550{ }^{\circ} \mathrm{C}\right]$, type of transition metal [Fe, Mn, Ag, Co, Cu, Ni], concentration of transition metal [0.05; $0.1 \mathrm{M}]$, metal deposition method [impregnation, ion exchange], post-treatment of the deposited metal [calcination under $\mathrm{O}_{2}$ flow, reduction under $\mathrm{H}_{2}$ flow], whereas among operating parameters, the type of VOC-Cl [perchlorethylene, chlorobenzene], concentration of Cl-VOCs [1.5; 9.5 umole $\mathrm{dm}^{-3}$ ], adsorption temperature [20; $\left.100^{\circ} \mathrm{C}\right]$, volumetric flow of Cl-VOCs [50; $\left.100 \mathrm{~cm}^{3} \mathrm{~min}^{-1}\right]$, mass of ZMt [0.15; $\left.0.3 \mathrm{~g}\right]$ are chosen. D-Optimal

\footnotetext{
1 Facultad de Ingeniería. Universidad Católica de la Santísima Concepción. Alonso de Ribera 2850. Concepción, Chile. E-mail: ariquelme@magister.ucsc.cl; hvaldes@ucsc.cl

* Autor de correspondencia
} 
design of experiment is used, and the number of experiments are reduced from 2.048 to 139. Adsorption experiments were carried out in a quartz U-shaped fixed bed adsorber, and the concentration of Cl-VOC was followed by gas chromatography. Experimental results are fitted to a second-order polynomial model. The analysis of variance (ANOVA) shows that the adjusted model is highly significant, with a satisfactory goodness of fit. ANOVA results also indicate that among the significant variables, modified zeolite samples by ion exchange using $\mathrm{Fe}$ or $\mathrm{Cu}$ increase the adsorption capacity toward $\mathrm{Cl}$-VOC. The concentration of $\mathrm{Cl}$-VOCs and the adsorption temperature show the biggest influence on the removal of $\mathrm{Cl}$-VOCs, among all the studied operating variables.

Keywords: Adsorption, ANOVA, Cl-VOCs, D-Optimal design, second-order model, modified zeolites.

\section{INTRODUCCIÓN}

Los compuestos orgánicos volátiles (COVs) son algunos de los contaminantes atmosféricos más comunes, cuyo origen va desde el humo producido por el tabaco hasta una amplia gama de industrias de fabricación de productos químicos, como: productos químicos orgánicos, plásticos, fibras sintéticas, pesticidas y productos farmacéuticos. Se generan también de los tratamientos de residuos peligrosos, en el almacenamiento y en la eliminación de desechos de las instalaciones industriales, en las industrias manufactureras de pintura y en las industrias papeleras [1-2].

El aumento de los niveles de contaminación atmosférica, debido a la presencia de COVs afecta directamente la salud de los seres vivos, provocando irritación de ojos y vías respiratorias, disminución de la función pulmonar y lesiones al hígado, riñones y sistema nervioso central [3]. Dentro de los COVs los Compuestos Orgánicos Volátiles Clorados (COVs-Cl) son considerados principalmente como xenobióticos, resistentes a la biodegradación y, por lo tanto, son muy persistentes en el medio ambiente [4]. La mayoría de los $\mathrm{COVs-Cl}$ son producidos por el hombre y son conocidos por ser irritantes, tóxicos, cancerígenos e inflamables [5]. Los COVs-Cl como el tetracloruro de carbono, tricloroetano, tricloroetileno, triclorotolueno pueden causar daños significativos al medio ambiente [6]. Estos son responsables de la producción de ozono troposférico, contribuyen en la eliminación del ozono en la estratosfera, además de la formación de smog fotoquímico [7].

La creciente conciencia ambiental ha puesto estrictas regulaciones para controlar las emisiones de COVs. En Chile existen leyes que regulan las emisiones de los COVs. El Decreto 594/1999 fija los valores máximos permisibles de los COVs en lugares de trabajo. Es por ello que la eliminación y reducción de los COVs es de suma importancia [1].

Existen varias técnicas disponibles para el control de emisiones de COVs-Cl, cada una presenta ventajas y limitaciones. Entre los procesos de tratamiento más convencionales para controlar las emisiones de COVs-Cl destacan la adsorción, la oxidación térmica, la oxidación catalítica y la biofiltración [1-2, 8-9]. La adsorción se ha utilizado ampliamente como un proceso alternativo fiable para abatir los COVs-Cl de las emisiones industriales, debido a la flexibilidad del sistema, al bajo consumo de energía y a los bajos costos de operación [10-12]. Los materiales microporosos como carbones activados, zeolitas sintéticas y naturales se han utilizado como adsorbentes de COVs-Cl, ya que su microporosidad actúa como reservorio de estos gases [13].

Por un lado, los carbones activados se seleccionan comúnmente como adsorbentes en muchos casos industriales, debido a sus altas capacidades de adsorción. Sin embargo, los carbones activados son relativamente caros y difíciles de regenerar debido a su sensibilidad a las altas temperaturas y la posibilidad de inflamarse [1, 14]. Por otra parte, las zeolitas sintéticas y naturales se han utilizado como adsorbentes para la eliminación de COVs-Cl de corrientes de gas residual [11, 15-17], siendo alternativas atractivas desde el punto de vista técnico y económico [7]. Las zeolitas tienen una alta estabilidad térmica [18], lo que les hace ser fáciles de regenerar y reutilizar para otros ciclos de proceso [19-20]. Lamentablemente, las bajas capacidades de adsorción de las zeolitas naturales hacia los COVs-Cl la hacen poco competitiva en comparación con carbones activados. Teniendo 
en cuenta esta limitación, las zeolitas naturales se pueden modificar químicamente con el fin de aumentar sus capacidades de adsorción hacia los $\mathrm{COVs-Cl}$. Resultados recientes muestran un aumento en la eliminación de compuestos orgánicos volátiles utilizando zeolita natural modificada con metales de transición de bajo costo, como $\mathrm{Co}, \mathrm{Cu}, \mathrm{Mn}$ y Fe [21-24]. Sin embargo, las variables más significativas que influyen en la modificación de las zeolitas naturales, así como tampoco las condiciones del proceso de adsorción en la eliminación de COVs-Cl han sido identificadas o modeladas.

Estudios actuales proponen el Diseño de Experimentos (DoE) como una metodología para la modelación de las variables operacionales [25-26], y en la identificación de la influencia de las variables de diseño [27-28]. EL DoE es una herramienta estadística utilizada para la construcción de un modelo empírico, que permite identificar si las variables de diseño o factores de entrada (cuantitativos o cualitativos), y las interacciones entre ellos, influyen en la variable dependiente (respuesta), por ejemplo la remoción de COVs [29].

Esta técnica no solo permite evaluar los efectos de interacción entre las variables seleccionadas, sino también reducir el número de corridas experimentales que deben llevarse a cabo, mediante la selección de algunas combinaciones de experimentos que transmitan la información necesaria sobre el conjunto, e indicando la manera de realizarse [30-31]. Uno de los enfoques más utilizados en el DoE, cuando existe un gran número de variables en estudio o cuando la región experimental es irregular, es el diseño D-Optimal, que maximiza el determinante de la matriz de información del diseño [30-31]. El D-Optimal permite mantener el número de experimentos tan bajo como sea posible, y selecciona la combinación más informativa de los factores [32]. Por lo tanto, el DoE del tipo D-Optimal es una solución eficaz y económica. En la literatura, se han informado distintas implementaciones del diseño D-Optimal para determinar los principales factores e investigar los cambios de la respuesta mediante la variación de cada factor [27, 32-34].

En este trabajo se realizan modificaciones químicas y térmicas a una zeolita natural chilena con el objetivo de mejorar la capacidad adsortiva. Por ello, el propósito de estudio que se presenta es identificar las variables que contribuyen en la modificación de la zeolita natural utilizando metales de transición (ZMt) y los parámetros operacionales más significativos que potencian el incremento en la capacidad de adsorción hacia COVs-Cl. Además, se evalúa el efecto de las interacciones entre las variables seleccionadas, estableciéndose los parámetros más influyentes durante la operación.

El presente trabajo es parte de un proyecto en desarrollo que investiga el uso de zeolitas naturales chilenas modificadas en la remoción de emisiones industriales de $\mathrm{COVs}-\mathrm{Cl}$ en un proceso combinado de adsorción y regeneración oxidativa con ozono gaseoso.

\section{METODOLOGÍA}

\section{Materiales}

La zeolita natural chilena (ZN) (53\% clinoptilonita, $40 \%$ modernita y $7 \%$ cuarzo) fue suministrada por la compañía chilena "Minera Formas" con un tamaño de partícula medio de 5,0 - 5,55 mm. Las muestras de zeolita utilizadas en el estudio se molieron y se tamizaron para obtener un tamaño de partícula en el rango de 0,300 - 0,425 mm. Luego se lavaron con agua desmineralizada, se secaron en estufa a $125^{\circ} \mathrm{C}$ por $24 \mathrm{~h}$, y se almacenaron en desecadores hasta su uso posterior. Los COVs-Cl, Percloroetileno (PERC) y Clorobenceno (CLB) fueron proveídos por la compañía MERCK (ambas con pureza (GC) $>99 \%$ ) en estado líquido. El agua desmineralizada fue obtenida desde el sistema EASY Pure ${ }^{\circledR}$ RF II.

Los metales de transición utilizados fueron adquiridos de la empresa MERCK en forma de sales de metales; nitrato de cobre $\left(\mathrm{Cu}\left(\mathrm{NO}_{3}\right)_{2} \times 3 \mathrm{H}_{2} \mathrm{O}\right)$, sulfato de manganeso $\left(\mathrm{MnSO}_{4} \times \mathrm{H}_{2} \mathrm{O}\right)$, nitrato de plata $\left(\mathrm{AgNO}_{3}\right)$, nitrato de cobalto $\left(\mathrm{Co}\left(\mathrm{NO}_{3}\right)_{2} \times 6 \mathrm{H}_{2} \mathrm{O}\right)$, nitrato de fierro $\left(\mathrm{Fe}\left(\mathrm{NO}_{3}\right)_{3} \times 9 \mathrm{H}_{2} \mathrm{O}\right)$ y nitrato de níquel $\left(\mathrm{Ni}\left(\mathrm{NO}_{3}\right)_{2} \times 6 \mathrm{H}_{2} \mathrm{O}\right)$, todos con pureza $>99 \%$. Los diferentes gases utilizados: Argón (Ar), Hidrógeno $\left(\mathrm{H}_{2}\right)$ y Aire Sintético $\left(\mathrm{N}_{2} / \mathrm{O}_{2}\right)$, todos de calidad extrapura $(99,99 \%)$ fueron suministrados por la empresa PRAXAIR Chile Ltda.

\section{Pretratamiento de la zeolita}

a. Intercambio iónico con amonio: $\mathrm{ZN}$ se le realizó un intercambio iónico con una solución de sulfato de amonio $\left[\left(\mathrm{NH}_{4}\right)_{2} \mathrm{SO}_{4}\right]\left(0,1 \mathrm{~mol} \mathrm{dm}^{-3}\right)$, relación de $1 \mathrm{~g}$ de $\mathrm{ZN}$ por $10 \mathrm{~cm}^{3}$ de solución, mediante 
agitación por $2 \mathrm{~h}$ a $27 \mathrm{rpm} \mathrm{y} 90{ }^{\circ} \mathrm{C}$, seguido de un lavado bajo agitación con agua desmineralizada por $2 \mathrm{~h}$, repitiendo 3 veces el procedimiento de lavado. Luego se secó a $125^{\circ} \mathrm{C}$ durante $24 \mathrm{~h}$. Las muestras se denominaron de acuerdo al número de intercambio iónico con amonio, como $\mathrm{ZN}_{\mathrm{W}}$, con W = 1 ó 2 veces realizado el procedimiento.

b. Desgasificación térmica: $\mathrm{ZN}_{\mathrm{w}}$ se desgasificó utilizando un flujo de argón $\left(100 \mathrm{~cm}^{3} \mathrm{~min}^{-1}\right)$ durante $2 \mathrm{~h}$ con una tasa de calentamiento de $10^{\circ} \mathrm{C} \mathrm{min}^{-1}$, en un reactor de lecho fijo de cuarzo en forma de $\mathrm{U}$ (4 mm de diámetro). Las muestras se denominaron de acuerdo a la temperatura de desgasificación como $\mathrm{ZN}_{\mathrm{W}}-\mathrm{Y}$, con $\mathrm{Y}=350^{\circ} \mathrm{C}$ o $550{ }^{\circ} \mathrm{C}$, respectivamente.

\section{Método de deposición del metal de transición}

Intercambio iónico: Las muestras de $\mathrm{ZN}_{\mathrm{W}}-\mathrm{Y}$ fueron modificadas por el método de intercambio iónico con las sales de los metales de transición $\mathrm{Mt}\left(\mathrm{Mt}=\mathrm{Cu}\left(\mathrm{NO}_{3}\right)_{2}, \mathrm{MnSO}_{4}, \mathrm{AgNO}_{3}, \mathrm{Co}\left(\mathrm{NO}_{3}\right)_{2}\right.$, $\left.\mathrm{Fe}\left(\mathrm{NO}_{3}\right)_{3}, \mathrm{Ni}\left(\mathrm{NO}_{3}\right)_{2}\right)$ en un equipo de agitación con baño termorregulado (modelo LSB-015S, Korea), utilizando soluciones de $0,1 \mathrm{~mol} \mathrm{dm}^{-3}$ de cada sal del Mt. El intercambio de iones entre $\mathrm{ZN}_{\mathrm{w}}-\mathrm{Y}$ y Mt fue llevado a cabo poniendo $10 \mathrm{~g} \mathrm{NH}_{4} \mathrm{Z}_{\mathrm{w}}-\mathrm{Y}$ en $100 \mathrm{~cm}^{3}$ de la solución de Mt, mediante agitación constante a $27 \mathrm{rpm}$, y $90{ }^{\circ} \mathrm{C}$, seguido de un lavado bajo agitación con agua desmineralizada por $2 \mathrm{~h}$, repitiendo 3 veces el procedimiento de lavado. El intercambio de iones con $0,05 \mathrm{~mol} \mathrm{dm}^{-3}$ y $0,075 \mathrm{~mol}$ $\mathrm{dm}^{-3}$ de solución de Mt y $100 \mathrm{~g}$ de $\mathrm{ZN}_{\mathrm{w}}-\mathrm{Y}$ también se realizó, repitiendo el procedimiento anterior.

Impregnación: $\mathrm{ZN}_{\mathrm{w}}-\mathrm{Y}$ fueron impregnadas en un rotavapor con baño termoregulado (IKA RV 10 basic, Staufen, Alemania). Las soluciones de sales de metales Mt para las diferentes concentraciones, $0,1 \mathrm{~mol} \mathrm{dm}^{-3}, 0,075 \mathrm{~mol} \mathrm{dm}^{-3}$ y $0,05 \mathrm{~mol} \mathrm{dm}^{-3}$ se prepararon de la misma manera descrita en el epígrafe anterior. La impregnación entre $\mathrm{NH}_{4} \mathrm{Z}_{\mathrm{w}}-\mathrm{Y}$ y Mt fue llevada a cabo poniendo $10 \mathrm{~g} \mathrm{NH}_{4} \mathrm{Z}_{\mathrm{w}}-\mathrm{Y}$ en la solución de Mt, la cual se hizo rotar a 100 rpm por $2 \mathrm{~h}$ a $80^{\circ} \mathrm{C}$ con presión de vacío de $10 \mathrm{~cm}$ $\mathrm{Hg}$. Luego se hizo rotar por $1 \mathrm{~h}$ a presión de vacío de $40 \mathrm{~cm} \mathrm{Hg}$ hasta evaporar la solución.

Todas las muestras resultantes fueron secadas posteriormente a $125^{\circ} \mathrm{C}$ durante $24 \mathrm{~h}$. Las muestras se denominaron de acuerdo al tipo de método de deposición del metal de transición como $\mathrm{ZN}_{\mathrm{W}}-\mathrm{Y} /$
Mt-X, con X = intercambio iónico o impregnación, respectivamente.

\section{Post-tratamiento del metal depositado}

Algunas muestras de $\mathrm{ZN}_{\mathrm{W}}-\mathrm{Y} / \mathrm{Mt}-\mathrm{X}$ fueron nuevamente desgasificadas bajo un flujo de argón $\left(100 \mathrm{~cm}^{3} \mathrm{~min}^{-1}\right)$ con tasa de calentamiento de $1{ }^{\circ} \mathrm{C}$ $\mathrm{min}^{-1}$. Las restantes se calcinaron con una corriente de aire $\left(100 \mathrm{~cm}^{3} \mathrm{~min}^{-1}\right)$ con una tasa de calentamiento de $1{ }^{\circ} \mathrm{C} \mathrm{min}{ }^{-1}$. Algunas de las muestras calcinadas se sometieron a una reacción de reducción con una corriente de $\mathrm{H}_{2}$ al $5 \%$ en $\mathrm{Ar}\left(20 \mathrm{~cm}^{3} \mathrm{~min}^{-1}\right)$ con una tasa de calentamiento de $1^{\circ} \mathrm{C} \mathrm{min}{ }^{-1}$. La temperatura final de los tres post-tratamientos depende de la temperatura de desgasificación térmica de $\mathrm{ZN}_{\mathrm{W}} \mathrm{Y} /$ Mt $-X$, con $Y=350{ }^{\circ} \mathrm{C}$ o $550{ }^{\circ} \mathrm{C}$, respectivamente. Todos los post-tratamientos se realizaron en un adsorbedor de lecho fijo de cuarzo con forma de U.

\section{Procedimiento experimental de adsorción}

Los experimentos de adsorción de $\mathrm{COVs}-\mathrm{Cl}$ se llevaron a cabo en un adsorbedor de lecho fijo de cuarzo con forma de $\mathrm{U}$, de diámetro interior $4 \mathrm{~mm}$ y espesor de 1,04 mm. Al interior del adsorbedor se colocó la muestra $\mathrm{ZN}_{\mathrm{W}}-\mathrm{Y} / \mathrm{Mt}-\mathrm{X}$ post-tratada, donde se hace circular la corriente gaseosa contaminada con el COVs-Cl. La corriente contaminada con el $\mathrm{COV}$ s- $\mathrm{Cl}$ se generó en el burbujeador inmerso en el baño termorregulador. El COVs-Cl es arrastrado hacia el adsorbedor mediante Ar regulado por un controlador de flujo (ver Figura 1). La concentración de COVs-Cl a la salida del reactor fue medida por un cromatógrafo de gases, GC, (modelo Clarus 500 GC, Shleton, USA) con detector de ionización de llama (FID) conectado a un computador para la lectura de datos, como se informó en otra publicación [35]. La cantidad total de COVs-Cl adsorbido dinámicamente, $q\left(\mu \mathrm{mol} \mathrm{g}^{-1}\right)$ se obtuvo de la curva de ruptura, como sigue:

$$
q=\frac{F C_{C O V_{S}-C l_{\text {in }}}}{m} \int_{0}^{t_{s}}\left(1-\frac{C_{C O V_{S}-C l_{t}}}{C_{C O V_{S}}-C l_{\text {in }}}\right) D s t
$$

donde $m$ es la masa de zeolita (g), $F$ es la tasa de flujo volumétrico $\left(\mathrm{cm}^{3} \mathrm{~min}^{-1}\right), t_{s}$ es el tiempo necesario para alcanzar la saturación de la zeolita con COVs-Cl (min), y $\mathrm{C}_{\mathrm{COVs}-\mathrm{Cl} \text {-in }}$ y $C_{C O V s-C l-\mathrm{t}}$ son la concentración del COVs-Cl seleccionado $\left(\mu \mathrm{mol} \mathrm{cm}{ }^{-3}\right)$ a la entrada y a la salida del adsorbedor en función del tiempo, respectivamente. 


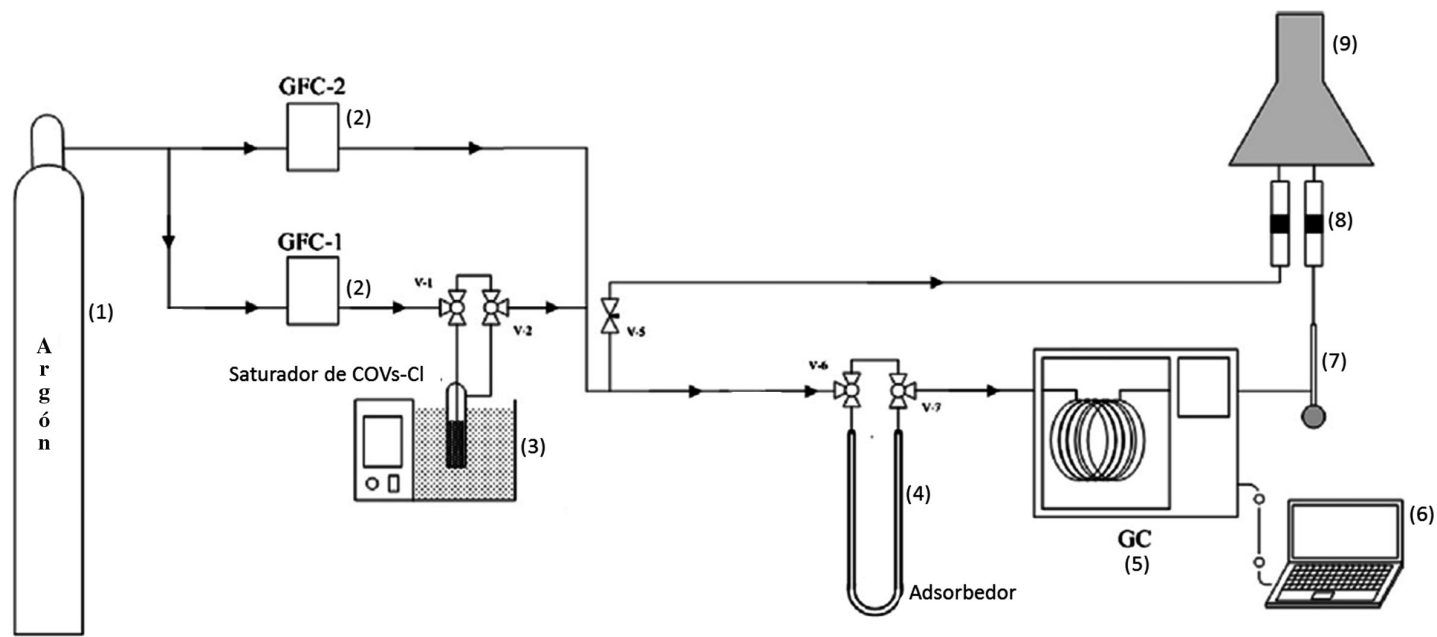

Figura 1. Esquema del equipo experimental utilizado [23]: (1) cilindro de Argón, (2) controlador de flujo másico (GFC), (3) saturador de COvs-Cl, (4) adsorbedor, (5) cromatógrafo de gases, (6) computador para registro de datos, (7) flujómetro, (8) trampas contenedoras de COVs-Cl residual, (9) extractor.

\section{Diseño de experimentos}

El DoE es la mejor manera para investigar el efecto de varias variables [36, 37], mediante la modelación de los parámetros dentro de los rangos especificados de estudio, ajustando una ecuación de predicción para la variable respuesta [37-38].

El diseño DoE del tipo D-Optimal con once factores (cinco cualitativos y seis cuantitativos) se utilizó para determinar los parámetros de preparación de la zeolita natural modificada con metal de transición (ZMt) y las condiciones del proceso de operación que aumentan la capacidad de adsorción hacia los COVs-Cl. Las variables del diseño experimental seleccionadas en la modificación de la zeolita natural, junto con las variables de proceso de operación para la eliminación de COVs-Cl se muestran en la Tabla 1.

Las variables de diseño estudiadas en la modificación de la zeolita natural para la remoción de los COVs$\mathrm{Cl}$ fueron: cantidad de intercambios iónicos $\left(x_{1}\right)$ temperatura de desgasificación $\left(x_{2}\right)$, tipo de metal de transición $\left(x_{3}\right)$, concentración del metal de

Tabla 1. Nivel experimental de las variables independientes.

\begin{tabular}{|c|c|c|c|c|}
\hline \multirow{2}{*}{ Variables } & \multirow{2}{*}{ Símbolo } & \multicolumn{3}{|c|}{ Nivel } \\
\hline & & Bajo & Medio & Alto \\
\hline Intercambio iónico con amonio & $\left(x_{1}\right)$ & uno & - & dos \\
\hline Temperatura de desgasificación $\left({ }^{\circ} \mathrm{C}\right)$ & $\left(x_{2}\right)$ & 350 & - & 550 \\
\hline Tipo de metal de transición & $\left(x_{3}\right)$ & Ag Co & $\mathrm{CuFe}$ & $\mathrm{Mn} \quad \mathrm{Ni}$ \\
\hline Concentración metal de transición $\left(\mathrm{mol} \mathrm{dm}^{-3}\right)$ & $\left(x_{4}\right)$ & 0,05 & 0,075 & 0,1 \\
\hline Método de deposición del metal & $\left(x_{5}\right)$ & intercambio iónico & - & impregnación \\
\hline Post-tratamiento del metal depositado & $\left(x_{6}\right)$ & desgasificación & calcinación & reducción \\
\hline Tipo de COVs-Cl & $\left(x_{7}\right)$ & PERC & - & CLB \\
\hline Concentración de COVs-Cl $\left(\mu \mathrm{mol} \mathrm{dm}{ }^{-3}\right)$ & $\left(x_{8}\right)$ & 1,5 & 5,5 & 9,5 \\
\hline Temperatura de adsorción $\left({ }^{\circ} \mathrm{C}\right)$ & $\left(x_{9}\right)$ & 20 & 60 & 100 \\
\hline Flujo volumétrico del VOCs- $\mathrm{Cl}\left(\mathrm{cm}^{3} \mathrm{~min}^{-1}\right)$ & $\left(x_{10}\right)$ & 50 & 75 & 100 \\
\hline Masa del adsorbente $(\mathrm{g})$ & $\left(x_{11}\right)$ & 0,15 & 0,225 & 0,3 \\
\hline
\end{tabular}


transición $\left(x_{4}\right)$, método de deposición del metal $\left(x_{5}\right)$ y post-tratamiento del metal depositado $\left(x_{6}\right)$. Las variables operacionales consideradas fueron: tipo de COVs-Cl $\left(x_{7}\right)$, concentración de COVs-Cl $\left(x_{8}\right)$, temperatura de adsorción $\left(x_{9}\right)$, flujo volumétrico de COVs-Cl $\left(x_{10}\right)$ y masa del adsorbente $\left(x_{11}\right)$. Las variables se estudiaron a diferentes niveles. Los niveles de las variables investigadas en este estudio se dan en la Tabla 1.

Como se puede observar en la Tabla 1 se estudiaron todas las variables a diferentes niveles. Todas las variables fueron identificadas con un valor codificado, de modo que todas ellas afectan a la respuesta de manera más uniforme, siendo las unidades de los parámetros irrelevantes. Las variables $x_{1}, x_{5}$ y $x_{7}$ son variables del tipo cualitativas con dos niveles en estudio, y fueron codificadas para tomar 0 (nivel bajo) ó 1 (nivel alto). Las variables $x_{3}$ y $x_{6}$ son cualitativas con más de dos niveles en estudio, y se representaron con n-1 variables dummy o binarias, donde $\mathrm{n}$ es el número de niveles de cada variable en estudio. Las variables dummy pueden tomar 1 cuando el nivel en estudio es seleccionado o 0 en caso contrario. Cuando todas las variables dummy toman 0 , el nivel de referencia es seleccionada. Para el caso de la variable $x_{3}$, el nivel de referencia es el Co, y para la variable $x_{6}$ el nivel de referencia es la desgasificación.
La variable $x_{2}$ es cuantitativa y discreta en sus parámetros, y fue codificada para tomar -1 (nivel bajo) o 1 (nivel alto). Las variables cuantitativas $x_{4}$, $x_{8}, x_{9}, x_{10} \mathrm{y} x_{11}$ son continuas y fueron codificadas a un valor central considerado cero, obligando a que varíen entre -1 a 1 , normalizando de esta manera los parámetros de las variables. La ecuación utilizada fue:

$$
X=\frac{x-\left[x_{\max }+x_{\min }\right] / 2}{\left[x_{\max }+x_{\min }\right] / 2}
$$

donde $x$ es la variable natural, $X$ es la variable normalizada, $x_{\max }$ y $x_{\min }$ son los valores máximos y mínimos de la variable natural. En la Tabla 2 se muestran los rangos y niveles codificados de las variables independientes cualitativas y cuantitativas.

En el DoE D-Optimal aplicado, primero se definieron los niveles de los parámetros, luego se seleccionó un modelo de segundo orden para identificar las variables que influyen en la capacidad de adsorción de ZMt hacia los COVs-Cl, como sigue:

$$
q=\beta_{0}+\sum_{i=1}^{16} \beta_{i} X_{i}+\sum_{i=1}^{16} \beta_{i i} X_{i i}^{2}+\sum_{i=1}^{15} \sum_{j=i+1}^{16} \beta_{i j} X_{i} X_{j}+\varepsilon
$$

donde $q$ es la variable respuesta capacidad de adsorción de la ZMt en la remoción de COVs-Cl

Tabla 2. Niveles codificados y valores reales de las variables cualitativas y cuantitativas.

\begin{tabular}{|l|c|c|c|c|}
\hline \multirow{2}{*}{ Variables } & \multirow{2}{*}{ Símbolo } & \multicolumn{3}{c|}{ Nivel } \\
\cline { 3 - 5 } & & -1 & $\mathbf{0}$ & $\mathbf{1}$ \\
\hline Intercambio iónico con amonio & $\left(\mathrm{X}_{1}\right)$ & - & uno & dos \\
\hline Temperatura de desgasificación $\left({ }^{\circ} \mathrm{C}\right)$ & $\left(\mathrm{X}_{2}\right)$ & 350 & - & 550 \\
\hline Plata como metal seleccionado & $\left(\mathrm{X}_{3 \mathrm{Ag}}\right)$ & - & otro metal & $\mathrm{Ag}$ \\
\hline Cobre como metal seleccionado & $\left(\mathrm{X}_{3 \mathrm{Cu}}\right)$ & - & otro metal & $\mathrm{Cu}$ \\
\hline Hierro como metal seleccionado & $\left(\mathrm{X}_{3 \mathrm{Fe}}\right)$ & - & otro metal & $\mathrm{Fe}$ \\
\hline Manganeso como de metal seleccionado & $\left(\mathrm{X}_{3 \mathrm{Mn}}\right)$ & - & otro metal & Mn \\
\hline Níquel como de metal seleccionado & $\left(\mathrm{X}_{3 \mathrm{Ni}}\right)$ & - & otro metal & $\mathrm{Ni}$ \\
\hline Concentración del metal de transición $\left(\mathrm{mol} \mathrm{dm}^{-3}\right)$ & $\left(\mathrm{X}_{4}\right)$ & 0,05 & 0,075 & 0,1 \\
\hline Método de deposición del metal & $\left(\mathrm{X}_{5}\right)$ & - & impregnación & intercambio iónico \\
\hline Calcinación como post-tratamiento seleccionado & $\left(\mathrm{X}_{6 \mathrm{Ca}}\right)$ & - & otro post-tratamiento & calcinación \\
\hline Reducción como post-tratamiento seleccionado & $\left(\mathrm{X}_{6 \mathrm{Red}}\right)$ & - & otro post-tratamiento & reducción \\
\hline Tipo de COVs-Cl & $\left(\mathrm{X}_{7}\right)$ & - & CLB & PERC \\
\hline Concentración de COVs-Cl $\left(\mu m o l \mathrm{dm}^{-3}\right)$ & $\left(\mathrm{X}_{8}\right)$ & 1,5 & 5,5 & 9,5 \\
\hline Temperatura de adsorción $\left({ }^{\circ} \mathrm{C}\right)$ & $\left(\mathrm{X}_{9}\right)$ & 20 & 60 & 100 \\
\hline Flujo volumétrico de COVs-Cl $\left(\mathrm{cm}^{3} \mathrm{~min}^{-1}\right)$ & $\left(\mathrm{X}_{10}\right)$ & 50 & 75 & 100 \\
\hline Masa del adsorbente $(\mathrm{g})$ & $\left(\mathrm{X}_{11}\right)$ & 0,15 & 0,225 & 0,3 \\
\hline
\end{tabular}


$\left(\mu \mathrm{mol} \mathrm{g}{ }^{-1}\right)$. $\beta_{0}$ es el término constante y $\beta_{i}$ son los coeficientes de regresión de los parámetros lineales, $\beta_{i i}$ son los coeficientes de regresión de los parámetros de segundo orden y $\beta_{i j}$ los coeficientes de regresión de los parámetros de interacción; $X_{i}$ y $X_{j}$ los niveles de las variables experimentales codificadas y $\varepsilon$ es el error residual asociado a los experimentos El software de análisis de datos MODDE 9.1.1 (Umetrics Inc., San José, California, USA) se utilizó para el diseño D-Optimal y para ajustar los datos experimentales a la ecuación polinómica de segundo orden mediante una técnica de regresión lineal múltiple.

Para evaluar la significación y la adecuación del modelo polinomial de segundo orden ajustado, e identificar los niveles de las variables de estudio, se realizó un análisis de la varianza (ANOVA). Para identificar qué tan bien las variables de estudio describen la variación de los datos acerca de su media, se utilizó el valor- $F$ de Fisher. La calidad del modelo ajustado y lo bien que el modelo predice un valor de respuesta se evaluó mediante el coeficiente de determinación ajustado $\left(R_{\text {ajustado }}^{2}\right)$ y también por el coeficiente de determinación predicho $\left(R_{\text {predicho }}^{2}\right)$ [38]. La precisión adecuada del modelo y la raíz cuadrada media de error (RMSE) se utilizó para identificar la adecuación del modelo para navegar por el espacio de diseño y su capacidad para predecir la respuesta.

\section{RESULTADOS Y DISCUSIÓN}

El DoE D-Optimal se desarrolló con un modelo de regresión lineal de segundo orden equilibrado para las variables cualitativas, con dos repeticiones en los puntos centrales, que permite por un lado aleatorizar el orden de ejecución además de garantizar la existencia de independencia en las observaciones y evitar el efecto de la variabilidad externa. Como resultado se obtuvieron 139 corridas experimentales a realizar de un total de 2.048 experimentos posibles en el diseño. En la Tabla 3 se entrega el resumen del DoE seleccionado.

Tabla 3. Resumen del diseño D-Optimal.

\begin{tabular}{|l|c|}
\hline \multicolumn{1}{|c|}{ Diseño } & D-Optimal \\
\hline Tipo de modelo & de segundo orden \\
\hline Número de términos del modelo & 130 \\
\hline Número máximo de experimentos & 2.048 \\
\hline $\mathrm{N}=$ Experimentos del diseño & 139 \\
\hline $\begin{array}{l}\text { Estadísticas del diseño: log (Det. } \\
\left.\mathrm{X}^{\prime} \mathrm{X}\right)\end{array}$ & 212,205 \\
\hline
\end{tabular}

\section{Ajuste del modelo del diseño D-Optimal y análisis estadístico}

Realizados los 139 experimentos entregados por el DoE D-Optimal, los resultados experimentales se ajustaron a una ecuación polinómica de segundo orden (ecuación (3)) mediante la técnica de regresión lineal múltiple utilizando el software de análisis de datos MODDE 9.1.1 (Umetrics Inc., San José, California, USA. La Tabla 4 muestra los resultados entregados del análisis de varianza (ANOVA) del modelo de regresión lineal de segundo orden ajustado.

Para probar la bondad de ajuste del modelo se utilizó el coeficiente de determinación $R^{2}$. El valor del coeficiente de determinación es 0,998 . Como existen 130 términos en el modelo ajustado, el $R^{2}$ resulta poco confiable al incrementarse en forma artificial con cada término que se agrega al modelo, aunque sea un término que no contribuya en nada a la explicación de la respuesta [37-38]. Cuando sucede esto se prefiere el estadístico $R_{\text {ajustado debido }}^{2}$ a que corrige el $R^{2}$ para el tamaño de la muestra y el número de términos en el modelo. En este estudio, el coeficiente de determinación ajustado $\left(R^{2}{ }_{\text {ajustado }}\right.$

Tabla 4. Resultados del ANOVA para el modelo de regresión lineal de segundo orden ajustado.

\begin{tabular}{|l|c|c|c|c|c|}
\hline Fuente de variación & Suma de cuadrados & Grados de libertad & Media cuadrática & Valor- $\boldsymbol{F}$ & Valor- $\boldsymbol{p}$ \\
\hline Modelo & $97.343,8$ & 130 & 748,799 & $45,5774 \mathrm{a}$ & $<0,0001$ \\
\hline Residuos & 131,433 & 8 & 16,4292 & & \\
\hline Falta de ajuste & 131,113 & 7 & 18,7304 & 58,4155 & 0,100 \\
\hline Error puro & 0,32064 & 1 & 0,32064 & & \\
\hline Total & $97.475,2$ & 138 & 706,342 & & \\
\hline
\end{tabular}

$R^{2}=0,9986 ; R_{\text {ajustado. }}^{2}=0,977 ; R_{\text {predicho }}^{2}=0,78094$.

Precisión $=22,786 ; R M S E=4,053$.

${ }^{\mathrm{a}} \mathrm{F}_{0,05(130,8)}=45,5774>\mathrm{F}_{0,05(130,8) \text { tabla }}=2,965$. 
$=0,977)$ es alto, indicando la alta significancia del modelo, y un buen ajuste entre el modelo de regresión y los valores experimentales. La diferencia entre los valores del coeficiente de determinación ajustado $\left(R_{\text {ajustado }}^{2}\right.$ y el coeficiente de determinación predicho $\left(R_{\text {predicho }}^{2}\right)$ es de alrededor de 0,2 , lo que sugiere una buena predictibilidad del modelo ajustado. De acuerdo con los resultados mostrados en la Tabla 4, un valor de precisión de 22,786 revela una relación adecuada señal/ruido, señalando la fiabilidad de los datos experimentales. Una proporción señal/ruido mayor que 4 es deseable y adecuada e indica un modelo de discriminación adecuado [44]. En este trabajo, el RMSE de 4,053 muestra que el modelo de regresión lineal de segundo orden se ajusta muy bien a cada punto de la región experimental y es adecuado para apoyar el ajuste del modelo.

El valor- $F$ de Fisher es una medida estadísticamente válida que asegura si las variables en estudio describen adecuadamente la variación de los datos sobre su media, y si los efectos estimados de los factores son reales [24, 26]. El ANOVA del modelo de regresión lineal de segundo orden demuestra que el modelo es altamente significativo, según se puede apreciar en los resultados del valor- $F$ de Fisher $\left(F_{\text {modelo }}=\right.$ $45,577)$ con un valor de probabilidad muy baja (valor- $p=0,0001)$. Por otra parte, el valor- $F$ del modelo es mucho mayor que el valor- $F$ de tabla $\left(F_{0,05(130,8) \text { tabular }}=2,965\right)$ con un nivel del $5 \%$, lo que indica que las diferencias de tratamiento son altamente significativas. Además, la prueba de falta de ajuste del modelo, permitió verificar si el orden del modelo es el correcto y si el modelo se ajusta de manera adecuada a los datos [36]. Con el valor- $F$ de la Falta de ajuste $=58,4155$ se confirmó que la falta de ajuste no es significativa en relación al error puro cuando el valor- $p<0,05$. La insignificancia de la falta de ajuste indica buena previsibilidad.

Los factores más significativos del modelo de regresión (con un valor-p < 0,05) junto con la estimación del parámetro, de acuerdo al ANOVA, se dan en la Tabla 5. Los valores- $p$ fueron utilizados como una herramienta para comprobar el nivel de significancia de cada coeficiente estimado en el modelo y que son necesarios para inferir el patrón de las interacciones entre las variables de estudio [25-26].

Por un lado, los resultados experimentales muestran que entre las variables de diseño, la temperatura de desgasificación $\left(\mathrm{X}_{2}\right)$ y el método de deposición del metal $\left(\mathrm{X}_{5}\right)$ son los más significativos (ambos con valor- $p<0,0001)$ y que poseen los mayores valores absolutos de los coeficientes ajustados. Esto sugiere que la temperatura de desgasificación a $350^{\circ} \mathrm{C}$ y el método de deposición del metal de intercambio iónico, producen un alto efecto en la capacidad de adsorción de la ZMt. A su vez el tipo de metal $\left(\mathrm{X}_{3}\right)$ influye considerablemente en la remoción de los COVs-Cl. Todos los metales de transición son significativos (valor-p $<0,01)$. Sin embargo, afectan de manera diferente en la capacidad de adsorción de la ZMt. $\mathrm{El} \mathrm{Cu}$, el Fe y el Ni son los metales más influyentes.

Por otro lado, los resultados muestran que entre todas las variables de operación, la concentración de COVs-Cl $\left(\mathrm{X}_{8}\right)$ y la temperatura de adsorción $\left(\mathrm{X}_{9}\right)$ son altamente significativas en la capacidad de adsorción de Z-Mt (ambos con valor- $p<0,0001$ ). Una temperatura de adsorción de $20^{\circ} \mathrm{C}$ y una concentración superior a $9,5 \mu \mathrm{mol} \mathrm{\textrm {dm } ^ { - 3 }}$ son los niveles de las variables que producen los efectivos más significativos en la eliminación de los COVs-Cl. La variable tipo de $\mathrm{COVs}-\mathrm{Cl}\left(\mathrm{X}_{7}\right)$ a adsorber mediante la ZMt es significativa (valor- $p<0,01)$. Esto indicaría que dentro de los dos COVs-Cl estudiados, el PERC tiene mayor afinidad hacia zeolita ZMt.

El efecto de la interacción entre las variables en estudio sobre la variable de respuesta también se lista en la Tabla 5. Los resultados muestran que las interacciones que existen entre concentración del metal de transición y método de deposición del metal $\left(\mathrm{X}_{4} * \mathrm{X}_{5}\right)$, concentración del metal de transición y concentración de COVs-Cl $\left(\mathrm{X}_{4} * \mathrm{X}_{8}\right)$, concentración del metal de transición y temperatura de adsorción $\left(\mathrm{X}_{4} * \mathrm{X}_{9}\right)$ de manera respectiva, son altamente significativas con altos valores absolutos de los coeficientes ajustados, y todas con valor- $p<0,001$. Estos resultados sugieren que estas interacciones son importantes en la modificación de la zeolita para aumentar su capacidad de adsorción. Una menor concentración del metal de transición depositado por medio del intercambio iónico, muestran resultados más significativos en el aumento de la capacidad de adsorción de la zeolita cuando se emplean altas concentraciones de $\mathrm{COVs}-\mathrm{Cl}$ y una baja temperatura de adsorción $\left(20^{\circ} \mathrm{C}\right)$.

Existe una relación significativa entre las variables temperatura de desgasificación y tipo de COVs-Cl 
Tabla 5. Coeficientes de la regresión significativos $(\alpha=0,05)$.

\begin{tabular}{|c|c|c|c|c|c|}
\hline Variable & Coeficiente & Valor-p & Variable & Coeficiente & Valor-p \\
\hline Constante & 61,134 & $<0,0001$ & $\mathrm{X}_{3 \mathrm{Ni}} \mathrm{X}_{6 \mathrm{Red}}$ & 19,145 & 0,0005 \\
\hline $\mathrm{X}_{1}$ & 14,980 & 0,0272 & $\mathrm{X}_{3 \mathrm{Ni}} \mathrm{X}_{8}$ & $-8,160$ & 0,0157 \\
\hline$X_{2}$ & $-23,305$ & $<0,0001$ & $\mathrm{X}_{3 \mathrm{Cu}} \mathrm{X}_{4}$ & $-1,408$ & 0,0119 \\
\hline $\mathrm{X}_{3 \mathrm{Cu}}$ & 5,259 & $<0,0001$ & $\mathrm{X}_{3 \mathrm{Cu}} \mathrm{X}_{5}$ & $-22,772$ & $<0,0001$ \\
\hline $\mathrm{X}_{3 \mathrm{Mn}}$ & $-1,831$ & $<0,0001$ & $\mathrm{X}_{3 \mathrm{Cu}} \mathrm{X}_{6 \mathrm{Cal}}$ & 16,588 & 0,0051 \\
\hline$X_{3 \mathrm{Ag}}$ & $-14,688$ & 0,0001 & $\mathrm{X}_{3 \mathrm{Cu}} \mathrm{X}_{6 \mathrm{Red}}$ & 28,063 & 0,0417 \\
\hline $\mathrm{X}_{3 \mathrm{Fe}}$ & 1,804 & 0,0000 & $\mathrm{X}_{3 \mathrm{Cu}} \mathrm{X}_{8}$ & 2,410 & 0,0007 \\
\hline $\mathrm{X}_{3 \mathrm{Ni}}$ & 2,591 & 0,0082 & $\mathrm{X}_{3 \mathrm{Cu}} \mathrm{X}_{9}$ & $-1,679$ & 0,0093 \\
\hline $\mathrm{X}_{3 \mathrm{Co}}$ & $-6,865$ & 0,0090 & $\mathrm{X}_{3 \mathrm{Cu}} \mathrm{X}_{10}$ & $-8,284$ & 0,0003 \\
\hline$X_{5}$ & 10,172 & $<0,0001$ & $\mathrm{X}_{3 \mathrm{Cu}} \mathrm{X}_{11}$ & 11,034 & $<0,0001$ \\
\hline$X_{7}$ & 23,892 & 0,0173 & $\mathrm{X}_{3 \mathrm{Mn}} \mathrm{X}_{4}$ & 11,106 & 0,0014 \\
\hline $\mathrm{X}_{8}$ & 3,767 & $<0,0001$ & $\mathrm{X}_{3 \mathrm{Mn}} \mathrm{X}_{5}$ & $-25,420$ & 0,0001 \\
\hline $\mathrm{X}_{9}$ & $-3,036$ & $<0,0001$ & $\mathrm{X}_{3 \mathrm{Mn}} \mathrm{X}_{6 \mathrm{Red}}$ & 2,354 & 0,0156 \\
\hline $\mathrm{X}_{4} \mathrm{X}_{4}$ & 6,983 & 0,0155 & $\mathrm{X}_{3 \mathrm{Mn}} \mathrm{X}_{7}$ & $-16,225$ & 0,0046 \\
\hline $\mathrm{X}_{8} \mathrm{X}_{8}$ & $-20,199$ & $<0,0001$ & $\mathrm{X}_{3 \mathrm{Mn}} \mathrm{X}_{8}$ & 4,041 & 0,0012 \\
\hline $\mathrm{X}_{9} \mathrm{X}_{9}$ & $-6,717$ & 0,0003 & $\mathrm{X}_{3 \mathrm{Mn}} \mathrm{X}_{9}$ & $-6,076$ & 0,0001 \\
\hline $\mathrm{X}_{1} \mathrm{X}_{3 \mathrm{Cu}}$ & 15,672 & 0,0365 & $\mathrm{X}_{3 \mathrm{Mn}} \mathrm{X}_{10}$ & $-11,872$ & 0,0001 \\
\hline$X_{1} X_{3 A g}$ & 15,999 & 0,0335 & $\mathrm{X}_{3 \mathrm{Mn}} \mathrm{X}_{11}$ & $-11,805$ & $<0,0001$ \\
\hline $\mathrm{X}_{1} \mathrm{X}_{5}$ & $-12,989$ & 0,0004 & $\mathrm{X}_{3 \mathrm{Co}_{0}} \mathrm{X}_{4}$ & 9,106 & 0,0014 \\
\hline $\mathrm{X}_{1} \mathrm{X}_{6 \mathrm{Cal}}$ & $-21,460$ & 0,0030 & $\mathrm{X}_{3 \mathrm{Co}} \mathrm{X}_{7}$ & $-8,225$ & 0,0046 \\
\hline $\mathrm{X}_{1} \mathrm{X}_{6 \text { Red }}$ & $-21,874$ & 0,0086 & $\mathrm{X}_{3 \mathrm{Co}} \mathrm{X}_{9}$ & 0,243 & 0,0032 \\
\hline $\mathrm{X}_{1} \mathrm{X}_{7}$ & $-9,205$ & 0,0027 & $\mathrm{X}_{3 \mathrm{Co}} \mathrm{X}_{10}$ & $-1,124$ & 0,0021 \\
\hline $\mathrm{X}_{1} \mathrm{X}_{8}$ & 2,918 & 0,0267 & $\mathrm{X}_{3 \mathrm{Co}} \mathrm{X}_{11}$ & 9,753 & $<0,0001$ \\
\hline $\mathrm{X}_{1} \mathrm{X}_{9}$ & 2,996 & 0,0155 & $\mathrm{X}_{4} \mathrm{X}_{5}$ & 9,543 & 0,0004 \\
\hline $\mathrm{X}_{1} \mathrm{X}_{10}$ & $-9,687$ & $<0,0001$ & $\mathrm{X}_{4} \mathrm{X}_{8}$ & $-6,484$ & $<0,0001$ \\
\hline$X_{1} X_{11}$ & $-3,421$ & 0,0115 & $\mathrm{X}_{4} \mathrm{X}_{9}$ & 6,938 & $<0,0001$ \\
\hline $\mathrm{X}_{2} \mathrm{X}_{3 \mathrm{Mn}}$ & $-7,997$ & 0,0092 & $\mathrm{X}_{4} \mathrm{X}_{10}$ & 3,071 & 0,0011 \\
\hline $\mathrm{X}_{2} \mathrm{X}_{3 \mathrm{Fe}}$ & 14,731 & 0,0005 & $\mathrm{X}_{4} \mathrm{X}_{11}$ & 1,370 & 0,0423 \\
\hline $\mathrm{X}_{2} \mathrm{X}_{4}$ & 5,871 & 0,0006 & $\mathrm{X}_{5} \mathrm{X}_{6 \mathrm{Cal}}$ & $-23,998$ & 0,0001 \\
\hline $\mathrm{X}_{2} \mathrm{X}_{5}$ & 10,948 & 0,0006 & $\mathrm{X}_{5} \mathrm{X}_{6 \mathrm{Red}}$ & $-18,745$ & 0,0240 \\
\hline $\mathrm{X}_{2} \mathrm{X}_{6 \mathrm{Cal}}$ & 13,287 & 0,0070 & $\mathrm{X}_{5} \mathrm{X}_{7}$ & $-25,099$ & $<0,0001$ \\
\hline $\mathrm{X}_{2} \mathrm{X}_{7}$ & $-23,153$ & $<0,0001$ & $\mathrm{X}_{5} \mathrm{X}_{8}$ & $-5,117$ & 0,0021 \\
\hline $\mathrm{X}_{2} \mathrm{X}_{8}$ & 5,773 & 0,0033 & $\mathrm{X}_{5} \mathrm{X}_{9}$ & $-3,515$ & 0,0362 \\
\hline $\mathrm{X}_{2} \mathrm{X}_{9}$ & $-3,560$ & 0,0202 & $\mathrm{X}_{5} \mathrm{X}_{10}$ & $-7,318$ & 0,0001 \\
\hline $\mathrm{X}_{3 \mathrm{Ag}} \mathrm{X}_{5}$ & $-0,569$ & 0,0456 & $\mathrm{X}_{6 \mathrm{Cal}} \mathrm{X}_{7}$ & 14,175 & 0,0046 \\
\hline $\mathrm{X}_{3 \mathrm{Ag}} \mathrm{X}_{6 \mathrm{Cal}}$ & 3,733 & 0,0002 & $\mathrm{X}_{6 \mathrm{Cal}} \mathrm{X}_{8}$ & 6,679 & 0,0411 \\
\hline $\mathrm{X}_{3 \mathrm{Ag}} \mathrm{X}_{6 \mathrm{Red}}$ & $-4,496$ & 0,0001 & $\mathrm{X}_{6 \mathrm{Cal}} \mathrm{X}_{10}$ & 1,302 & 0,0005 \\
\hline $\mathrm{X}_{3 \mathrm{Ag}} \mathrm{X}_{7}$ & 1,448 & 0,0251 & $\mathrm{X}_{6 \mathrm{Cal}} \mathrm{X}_{11}$ & $-5,153$ & 0,0039 \\
\hline $\mathrm{X}_{3 \mathrm{Ag}} \mathrm{X}_{9}$ & 8,551 & 0,0008 & $\mathrm{X}_{6 \text { Red }} \mathrm{X}_{8}$ & 8,261 & 0,0053 \\
\hline $\mathrm{X}_{3 \mathrm{Ag}} \mathrm{X}_{11}$ & 4,906 & 0,0065 & $\mathrm{X}_{6 \mathrm{Red}} \mathrm{X}_{10}$ & $-12,286$ & $<0,0001$ \\
\hline $\mathrm{X}_{3 \mathrm{Fe}} \mathrm{X}_{4}$ & $-1,914$ & 0,0021 & $\mathrm{X}_{6 \mathrm{Red}} \mathrm{X}_{11}$ & 2,127 & 0,0047 \\
\hline $\mathrm{X}_{3 \mathrm{Fe}} \mathrm{X}_{5}$ & 15,385 & 0,0005 & $\mathrm{X}_{7} \mathrm{X}_{9}$ & $-10,112$ & $<0,0001$ \\
\hline $\mathrm{X}_{3 \mathrm{Fe}} \mathrm{X}_{6 \mathrm{Cal}}$ & $-17,378$ & 0,0005 & $\mathrm{X}_{7} \mathrm{X}_{10}$ & $-3,085$ & 0,0400 \\
\hline $\mathrm{X}_{3 \mathrm{Fe}} \mathrm{X}_{6 \mathrm{Red}}$ & 28,666 & $<0,0001$ & $\mathrm{X}_{7} \mathrm{X}_{11}$ & $-8,876$ & $<0,0001$ \\
\hline $\mathrm{X}_{3 \mathrm{Fe}} \mathrm{X}_{8}$ & $-18,568$ & $<0,0001$ & $\mathrm{X}_{8} \mathrm{X}_{9}$ & 3,025 & 0,0024 \\
\hline $\mathrm{X}_{3 \mathrm{Fe}} \mathrm{X}_{9}$ & 12,410 & 0,0001 & $\mathrm{X}_{9} \mathrm{X}_{10}$ & 2,286 & 0,0063 \\
\hline $\mathrm{X}_{3 \mathrm{Fe}} \mathrm{X}_{10}$ & 14,098 & $<0,0001$ & $\mathrm{X}_{9} \mathrm{X}_{11}$ & 2,304 & 0,0040 \\
\hline $\mathrm{X}_{3 \mathrm{Ni}} \mathrm{X}_{5}$ & 1,880 & 0,0077 & $\mathrm{X}_{10} \mathrm{X}_{11}$ & $-4,006$ & 0,0007 \\
\hline $\mathrm{X}_{3 \mathrm{Ni}} \mathrm{X}_{6 \mathrm{Cal}}$ & $-13,957$ & 0,0003 & & & \\
\hline
\end{tabular}


$\left(\mathrm{X}_{2} * \mathrm{X}_{7}\right)$, método de deposición del metal y tipo de COVs- $\mathrm{Cl}\left(\mathrm{X}_{5} * \mathrm{X}_{7}\right)$, tipo de COVs-Cl y temperatura de adsorción $\left(\mathrm{X}_{7} * \mathrm{X}_{9}\right)$, todas con valor- $p<0,0001$ respectivamente. Es posible inferir que estas interacciones permiten mejorar la capacidad de remoción del COVs-Cl. El PERC es removido en mayor cantidad cuando a la zeolita natural se le realiza un intercambio iónico con el metal de transición, desgasificada a $350{ }^{\circ} \mathrm{C}$, y con una temperatura de adsorción de $20^{\circ} \mathrm{C}$.

Las interacciones entre las variables concentración de COVs-Cl y temperatura de adsorción $\left(\mathrm{X}_{8} * \mathrm{X}_{9}\right)$, así como flujo volumétrico del COVs-Cl y masa de la $\mathrm{ZMt}\left(\mathrm{X}_{10} * \mathrm{X}_{11}\right)$, son importantes en la mejora de la capacidad de adsorción de la zeolita modificada para eliminar COVs-Cl a partir de corrientes contaminadas. Además se aprecia que dichas relaciones son significativas, todas con valor- $p$ $<0,001$. Una mayor masa de la ZMt y un menor flujo volumétrico del COVs-Cl pueden ser las mejores condiciones experimentales para la adsorción de los COVs-Cl mediante la zeolita modificada con metales de transición.

La relación existente entre las variables tipo de metal de transición con las restantes variables en estudio, poseen una efecto diferenciado según el tipo de metal seleccionado, de acuerdo a los valores respectivos que se muestran en la Tabla 5. Entre los metales más influyentes, el cobre $(\mathrm{Cu})$ posee una relación significativa (todos los valores- $p<0,001$ ) con las variables de operación $\left(\mathrm{X}_{3 \mathrm{Cu}} * \mathrm{X}_{8}, \mathrm{X}_{3 \mathrm{Cu}} * \mathrm{X}_{9}, \mathrm{X}_{3 \mathrm{Cu}} * \mathrm{X}_{10}\right.$, $\mathrm{X}_{3 \mathrm{Cu}} * \mathrm{X}_{11}$ ), lo que sugiere que estas interacciones son importantes en la zeolita modificada con $\mathrm{Cu}$ para aumentar su capacidad de adsorción. El Fe a su vez, posee una relación significativa (valores- $p$ $<0,001)$ con la mayoría de las variables de diseño $\left(\mathrm{X}_{3 \mathrm{Fe}} * \mathrm{X}_{4}, \mathrm{X}_{3 \mathrm{Fe}} * \mathrm{X}_{5}, \mathrm{X}_{3 \mathrm{Fe}} * \mathrm{X}_{6 \mathrm{Cal}}, \mathrm{X}_{3 \mathrm{Fe}} * \mathrm{X}_{6 \mathrm{Red}}\right)$, lo que permite inferir que estas interacciones mejoran la capacidad de remoción del COVs-Cl cuando la zeolita natural es modificada con el Fe.

\section{Comprobación de la adecuación del modelo ajustado}

El análisis de los datos del modelo debe ser validado por un análisis de comprobación de idoneidad para asegurar que el modelo representa con precisión el sistema real o de lo contrario el análisis puede dar resultados pobres o engañosos [36-39]. Esto consiste en comprobar que se cumplan los supuestos del modelo: normalidad, varianza constante (homocedasticidad) e independencia. Estos supuestos se pueden traducir en supuestos sobre el término error en el modelo, definido como $e_{i}=Y_{i}-\hat{Y}_{i}, \mathrm{i}=1,2 \ldots, \mathrm{k}$, donde $\mathrm{k}$ es el número de experimentos [36-37].

En la Figura 2 se muestra la probabilidad normal de los residuales studentizados asociados a los experimentos, permitiendo detectar valores atípicos y evaluar la normalidad de los residuos.

El gráfico de probabilidad normal indica que el error del experimento $\mathrm{N}^{\circ} 15$ superó la varianza residual (más de 4 unidades de desviación estándar), considerándose un valor atípico. Los demás residuales se ajustan a una línea recta, lo que indica que se distribuyen normalmente, además de una excelente adecuación del modelo de regresión utilizado.

Para corroborar el análisis de que los errores se distribuyen normal, se realizó la prueba no paramétrica de Kolmogorov Smirnov [40,41], que contrasta la hipótesis de que los errores se ajustan a una distribución normal con media cero y varianza constante. El resultado de la prueba estadística K-S (valor- $p=0,763$ y Estadístico K-S = 0,669) indica que los errores si siguen una distribución normal, de manera tal que la inferencia antes mencionada es válida. Esto demuestra, además, que los valores experimentales están en buen acuerdo con los valores previstos.

En la Figura 3 se muestran los residuos studentizados en función de la cantidad de COVs-Cl adsorbida por la ZMt $\left(\mu \mathrm{mol} \mathrm{g}^{-1}\right)$ predicha por el modelo ajustado.

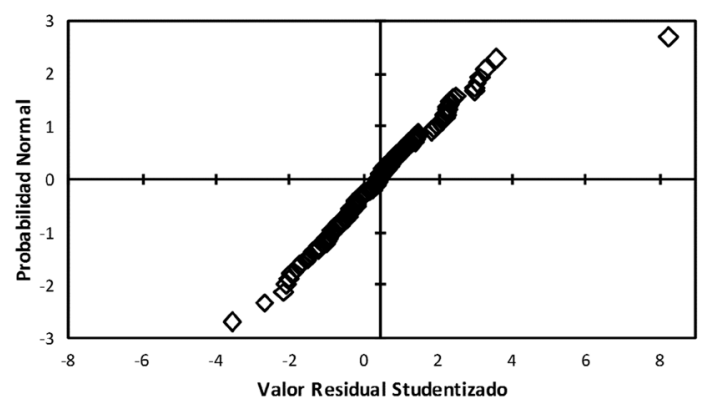

Figura 2. Probabilidad normal de los residuos experimentales. 


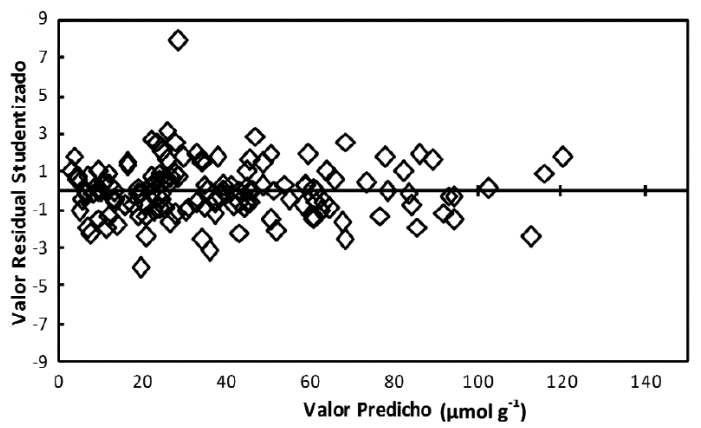

Figura 3. Residuos studentizados v/s. capacidad de adsorción de la ZMt predicha.

Como se puede observar, los residuales exhiben una ligera tendencia a formar un patrón en forma de embudo, que podría significar que la varianza de las observaciones originales no es constante para todos los valores de respuesta.

Con el fin de comprobar si la hipótesis de homocedasticidad se satisface, se aplicó la prueba de Levene [42] que contrasta la hipótesis de que los errores poseen varianza constaste. Los resultados de la prueba estadística de Levene (valor $-p=$ 0,083 y Estadístico de Levene $=3,874$ ) señalan que no existe evidencia suficiente para rechazar la hipótesis en estudio, cumpliéndose el supuesto de homocedasticidad de los errores.

La Figura 4 muestra los residuales studentizados y el orden en que se ejecutaron los experimentos, permitiendo detectar cualquier tipo de dependencia de los residuos. Al no observar una tendencia o patrón no aleatorio de los residuos, esto es evidencia de que no existe una correlación entre los errores, y por lo tanto, se asume el supuesto de independencia de los errores [29].

La Figura 5 muestra la relación entre el valor real y el valor predicho de la remoción de COVs-Cl por la zeolita modificada con metales de transición. Los valores reales se obtuvieron de las pruebas experimentales y los valores de predicción se determinaron utilizando el modelo polinomial de segundo orden obtenido con el diseño de experimentos D-Optimal. Dado que los valores se ajustan a la línea recta, y como el $R^{2}$ y el $R^{2}{ }_{a d j}$. poseen valores altos, el modelo muestra un buen ajuste.

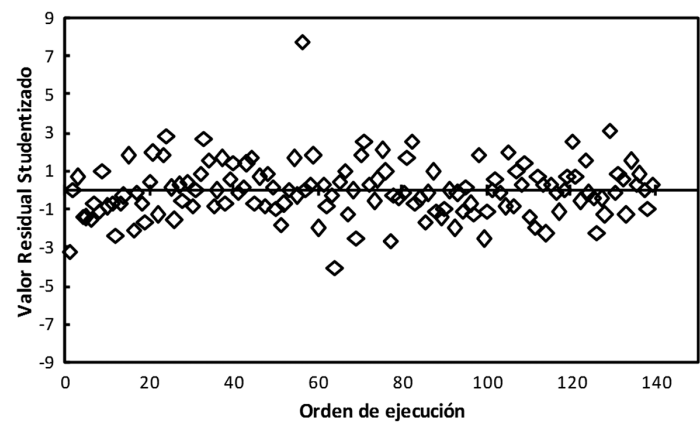

Figura 4. Los residuos en relación al orden de ejecución de los experimentos.

Verificación experimental de los resultados predichos por el modelo de regresión ajustado. Para comprobar el análisis estadístico de los factores más significativos del modelo de regresión ajustado, que son necesarios para comprender el patrón de las interacciones entre las variables de estudio, se analizó el 10\% de los mayores niveles de la capacidad de adsorción de COVs-Cl obtenidos sobre zeolitas naturales chilenas modificadas.

En la Tabla 6 se aprecia que del total de experimentos realizados, las mayores capacidades de adsorción oscilan entre los 121 y $84 \mu \mathrm{mol} \mathrm{g}{ }^{-1}$. Los niveles de las variables en estudio que tienen en común los experimentos con mayores capacidad de adsorción cuentan con la temperatura de desgasificación de $350{ }^{\circ} \mathrm{C}$, el método de intercambio iónico como deposición del metal, una temperatura de adsorción de $20{ }^{\circ} \mathrm{C}$, utilizando un flujo volumétrico de $100 \mathrm{~cm}^{3} \mathrm{~min}^{-1}$. Los metales más influyentes en la modificación de la zeolita natural fueron el $\mathrm{Cu}$ y el Fe. Se observa, además, una mayor capacidad

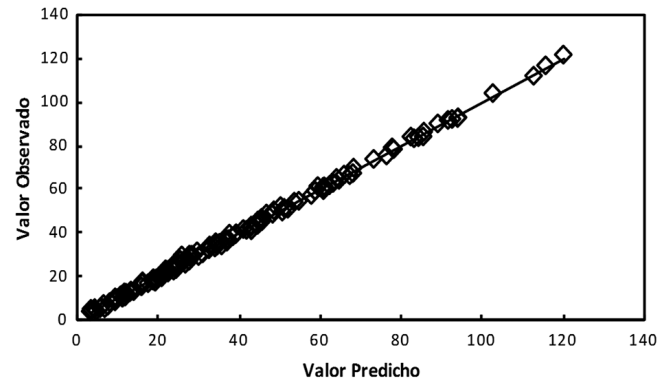

Figura 5. Valor observado v/s. valor predicho de la capacidad de adsorción de la ZMt. 
Tabla 6. Variable respuesta y variables de los mejores experimentos del DoE D-Optimal.

\begin{tabular}{|c|c|c|c|c|c|c|c|c|c|c|c|}
\hline $\begin{array}{c}\text { Variable } \\
\text { Respuesta } \\
\left(\text { umol g }^{-1}\right)\end{array}$ & $X_{1}$ & $\begin{array}{c}\mathrm{X}_{2} \\
\left({ }^{\circ} \mathrm{C}\right)\end{array}$ & $\begin{array}{c}\mathrm{X}_{3} \\
\left(\mathrm{~mol} \mathrm{dm^{-3 }}\right)\end{array}$ & $X_{4}$ & $X_{5}$ & $X_{6}$ & $\mathbf{X}_{7}$ & $\begin{array}{c}\mathbf{X}_{8} \\
\left(\mu m o l d m^{-3}\right)\end{array}$ & $\begin{array}{c}\mathrm{X}_{\mathbf{9}} \\
\left({ }^{\circ} \mathrm{C}\right)\end{array}$ & $\begin{array}{c}\mathbf{X}_{10} \\
\left(\mathrm{~cm}^{3} \mathrm{~min}^{-1}\right)\end{array}$ & $\begin{array}{l}X_{11} \\
(g)\end{array}$ \\
\hline 121,37 & dos & 350 & $\mathrm{Cu}$ & 0,1 & intercambio & calcinación & PERC & 9,5 & 20 & 100 & 0,3 \\
\hline 116,45 & uno & 350 & $\mathrm{Fe}$ & 0,1 & impregnación & desgasificación & PERC & 1,5 & 100 & 75 & 0,3 \\
\hline 111,71 & uno & 350 & $\mathrm{Fe}$ & 0,1 & intercambio & calcinación & PERC & 1,5 & 100 & 100 & 0,15 \\
\hline 99,37 & dos & 350 & $\mathrm{Fe}$ & 0,1 & intercambio & reducción & CLB & 9,5 & 100 & 100 & 0,3 \\
\hline 93,70 & dos & 350 & $\mathrm{Cu}$ & 0,05 & intercambio & desgasificación & PERC & 9,5 & 100 & 50 & 0,15 \\
\hline 93,67 & uno & 350 & $\mathrm{Ni}$ & 0,05 & intercambio & calcinación & PERC & 9,5 & 20 & 100 & 0,3 \\
\hline 91,92 & dos & 350 & $\mathrm{Ag}$ & 0,05 & intercambio & reducción & PERC & 9,5 & 20 & 50 & 0,225 \\
\hline 91,49 & uno & 350 & $\mathrm{Cu}$ & 0,075 & intercambio & calcinación & PERC & 1,5 & 20 & 50 & 0,15 \\
\hline 89,87 & uno & 550 & $\mathrm{Fe}$ & 0,075 & intercambio & reducción & PERC & 5,5 & 60 & 75 & 0,225 \\
\hline 86,10 & dos & 350 & Co & 0,05 & intercambio & desgasificación & CLB & 9,5 & 20 & 100 & 0,15 \\
\hline 83,94 & uno & 350 & $\mathrm{Cu}$ & 0,05 & intercambio & reducción & CLB & 9,5 & 60 & 100 & 0,15 \\
\hline 83,59 & dos & 350 & $\mathrm{Ag}$ & 0,1 & intercambio & calcinación & PERC & 9,5 & 20 & 100 & 0,15 \\
\hline 83,58 & uno & 350 & $\mathrm{Fe}$ & 0,05 & impregnación & reducción & PERC & 9,5 & 20 & 100 & 0,15 \\
\hline
\end{tabular}

de adsorción de la zeolita modificada con metal de transición hacia el percloroetileno.

La mayor capacidad de adsorción de la ZMt en la remoción de COVs-Cl $\left(\mu \mathrm{mol} \mathrm{g}{ }^{-1}\right)$ que entregaron los resultados experimentales realizados según la planificación del DoE D-Optimal, fue de $121 \mu \mathrm{mol}$ $\mathrm{g}^{-1}$ de percloroetileno. Esta capacidad de adsorción se obtiene usando zeolita natural modificada-Cu (Z-Cu). La muestra de $\mathrm{Z}-\mathrm{Cu}$ es generada con un pre-tratamiento de dos intercambios iónicos con sal de amonio, seguido por una desgasificación térmica de $350{ }^{\circ} \mathrm{C}$. Donde los iones de $\mathrm{Cu}^{2+}$ se depositaron mediante intercambio iónico $(0,1 \mathrm{~mol}$ $\mathrm{dm}^{-3}$ ) y posteriormente se calcinó con un flujo de oxígeno a $350^{\circ} \mathrm{C}$. Las condiciones más influyentes de adsorción resultan ser: $100 \mathrm{~cm}^{3} \mathrm{~min}^{-1}$ de flujo volumétrico, 9,5 $\mathrm{mol} \mathrm{dm}^{-3}$ de la concentración de percloroetileno, usando una masa de $0,3 \mathrm{~g}$, a $20^{\circ} \mathrm{C}$.

Para corroborar el aporte del metal de transición utilizado en la modificación de la zeolita natural que incrementa la capacidad de adsorción se realizó el procedimiento experimental de adsorción, empleando los niveles de las variables de la mayor capacidad de adsorción encontrada por el DoE D-Optimal, sin incluir la etapa de deposición del metal de transición (ver Tabla 7). El resultado de la capacidad de adsorción de la zeolita natural es de $69 \mu \mathrm{mol} \mathrm{g}{ }^{-1}$ de percloroetileno, lo que muestra que solo un $43 \%$ de la capacidad de adsorción es alcanzada cuando se utiliza la zeolita natural sin modificación en comparación con la zeolita modificada con metal de transición. De esta manera, se evidencia una mejora considerable en la capacidad de adsorción incluyendo el metal de transición en la modificación de la zeolita natural.

La mayor capacidad de adsorción de la ZMt en la remoción de clorobenceno $\left(\mu \mathrm{mol} \mathrm{g} \mathrm{g}^{-1}\right)$ que entregaron los resultados experimentales entregados por el DoE D-Optimal fue de $99 \mu \mathrm{mol} \mathrm{g}{ }^{-1}$. Esta capacidad de adsorción se obtiene usando zeolita natural modificada$\mathrm{Fe}(\mathrm{Z}-\mathrm{Fe})$. La muestra de Z-Fe es generada con un pretratamiento de dos intercambios iónicos con sal de amonio, seguido por una desgasificación térmica de $350^{\circ} \mathrm{C}$. Donde los iones de $\mathrm{Fe}^{3+}$ se depositaron mediante intercambio iónico $\left(0,1 \mathrm{~mol} \mathrm{dm}^{-3}\right) \mathrm{y}$, posteriormente, realizó una reducción con una corriente de $\mathrm{H}_{2}$ al $5 \%$ en Argón. Las condiciones más influyentes de adsorción resultan ser: $100 \mathrm{~cm}^{3} \mathrm{~min}^{-1}$ de flujo volumétrico, 9,5 mol dm${ }^{-3}$ de la concentración de clorobenceno, usando una masa de $0,3 \mathrm{~g}$, a $100^{\circ} \mathrm{C}$.

Para corroborar cuál es el aporte del metal en la modificación de la zeolita natural se realizó el procedimiento experimental de adsorción de clorobenceno utilizando los mismos niveles descritos anteriormente, sin la deposición de los iones de $\mathrm{Fe}^{3+}$ mediante intercambio iónico, entregando una capacidad de adsorción de $45 \mu \mathrm{mol} \mathrm{g}{ }^{-1}$, produciendo una disminución del $54 \%$ en la capacidad de adsorción de la zeolita sin modificación (ver Tabla 7). De esta 
Tabla 7. Capacidad de adsorción de la zeolita modificada v/s zeolita natural sin modificación.

\begin{tabular}{|l|c|c|c|c|}
\hline \multicolumn{1}{|c|}{ Muestra de zeolita } & $\mathbf{Z N}_{\mathbf{2}}$-350/Cu-Int-Cal & $\mathbf{Z N}$ & $\mathbf{Z N}_{\mathbf{2}}$-350/Fe-Int-Red & ZN \\
\hline Tipo de COVs-Cl & PERC & PERC & CLB & CLB \\
\hline Concentración de COVs-Cl $\left(\mu \mathrm{mol} \mathrm{dm}^{-3}\right)$ & 9,5 & 9,5 & 9,5 & 9,5 \\
\hline Temperatura de adsorción $\left({ }^{\circ} \mathrm{C}\right)$ & 20 & 20 & 100 & 100 \\
\hline Flujo volumétrico $\left(\mathrm{cm}^{3} \mathrm{~min}^{-1}\right)$ & 100 & 100 & 100 & 100 \\
\hline Masa del adsorbente $(\mathrm{g})$ & 0,3 & 0,3 & 0,3 & 0,3 \\
\hline Variable respuesta $\left(\boldsymbol{\mu} \mathbf{m o l ~}^{-1}\right)$ & $\mathbf{1 2 1 , 3 7}$ & $\mathbf{6 9 , 4 5}$ & $\mathbf{9 9 , 3 7}$ & $\mathbf{9 3 , 7}$ \\
\hline
\end{tabular}

manera se observa una mejora en la capacidad de adsorción incluyendo el metal de transición en la modificación de la zeolita natural.

Para analizar el comportamiento del clorobenceno bajo los mismos parámetros experimentales de la máxima capacidad de adsorción obtenida hacia el percloroetileno, es que se realizó la experimentación bajo las mismas condiciones. El resultado experimental de la capacidad de adsorción de la zeolita natural modificada con $\mathrm{Cu}$ es de 86 $\mu$ mol g ${ }^{-1}$ de clorobenceno, un $29 \%$ menor en la capacidad de adsorcion respecto al percloroetileno $\left(121 \mu \mathrm{mol} \mathrm{g}{ }^{-1}\right)$.

\section{CONCLUSIONES}

El diseño de experimentos del tipo D-Optimal por medio de un modelo de regresión lineal de segundo orden permitió identificar la región experimental que transmite la información necesaria sobre el conjunto de factores en estudio, logrando reducir considerablemente el número de corridas experimentales y la cantidad de recursos utilizados en la investigación.

Los resultados del análisis de varianza (ANOVA) mostraron un buen ajuste entre los valores predichos obtenidos por el modelo, en comparación con los resultados experimentales. Los coeficientes de correlación obtenidos del modelo $R^{2}$ y $R_{\text {ajus. }}^{2}$ fueron 0,9986 y 0,977 , respectivamente. $\mathrm{La}$ comprobación de la adecuación del modelo mediante la verificación de los supuestos del modelo: normalidad, homocedasticidad e independencia, fue satisfactoria.

Los resultados indican que las muestras de zeolita modificada por intercambio iónico utilizando $\mathrm{Cu}$ y $\mathrm{Fe}$, aumentan la capacidad de adsorción de la Z-Mt.
Entre los factores más importantes que influyen en capacidad de adsorción de la ZMt se encuentra la temperatura de desgasificación a $350{ }^{\circ} \mathrm{C}$ y el intercambio iónico como método de deposición del metal, ambos altamente significativos y con altos valores absolutos de sus coeficientes ajustados. La concentración del COVs-Cl y la temperatura de adsorción muestran la mayor influencia en la eliminación de compuestos orgánicos volátiles clorados, entre todas las variables de operación estudiadas.

La mayor capacidad de adsorción de ZMt en la remoción de COVs- $\mathrm{Cl}$ encontrados en esta investigación fue de $121 \mu \mathrm{mol} \mathrm{g}{ }^{-1}$ de percloroetileno. Esta capacidad de adsorción se obtiene usando zeolita natural modificada con cobre ( $\mathrm{Z}-\mathrm{Cu})$. La muestra de $\mathrm{Z}$-Cu es generada con un pretratamiento de dos intercambios iónicos con sal de amonio, seguido por una desgasificación térmica de $350^{\circ} \mathrm{C}$. Donde los iones de $\mathrm{Cu}^{2+}$ se depositaron mediante intercambio iónico $\left(0,1 \mathrm{~mol} \mathrm{dm}^{-3}\right)$ y, posteriormente, se calcinó con un flujo de oxígeno a $350^{\circ} \mathrm{C}$. Las condiciones más influyentes en la adsorción resultan ser: $100 \mathrm{~cm}^{3} \mathrm{~min}^{-1}$ de flujo volumétrico, $9,5 \mathrm{~mol} \mathrm{dm}^{-3}$ de la concentración de percloroetileno, usando una masa de $0,3 \mathrm{~g}$, a $20^{\circ} \mathrm{C}$.

El efecto del tipo de COVs-Cl utilizado sobre la capacidad de adsorción de la ZMt es altamente importante. Bajo los mismos parámetros experimentales de máxima capacidad de adsorción obtenidas con el percloroetileno, el resultado de la capacidad de adsorción de clorobenceno fue de $86 \mu \mathrm{mol} \mathrm{g} \mathrm{g}^{-1}$, demostrando la alta influencia de la naturaleza química del COVs-Cl sobre la capacidad de adsorción de la zeolita modificada con el metal de transición.

La selección del tipo de metal de transición en la modificación de la zeolita natural para aumentar su 
capacidad de adsorción hacia los COVs-Cl resultó ser una variable crucial. Los resultados experimentales mostraron que cuando se utiliza zeolita natural sin modificaciones la capacidad de adsorción hacia el percloroetileno y clorobenceno solo alcanzó un $43 \%$ y 54\% de la capacidad de adsorción de las zeolitas modificadas con $\mathrm{Cu}$ y $\mathrm{Fe}$, respectivamente.

El diseño de experimentos D-Optimal mostró ser una herramienta efectiva en la identificación de las variables más significativas en la modificación de zeolita natural chilena con metales de transición, y en el reconocimiento de los parámetros operacionales más importantes del proceso de adsorción de COVs-Cl. También permitió establecer los efectos más significativos de las interacciones entre las variables estudiadas.

\section{AGRADECIMIENTOS}

Los autores agradecen a la Comisión Nacional de Investigación Científica y Tecnológica (CONICYT) de Chile, Fondo Nacional de Desarrollo Científico y Tecnológico de Chile (FONDECYT) con el Proyecto (CONICYT/FONDECYT Regular N ${ }^{\circ} 1130560$ ) por el apoyo brindado en el desarrollo de la investigación.

El Sr. Riquelme manifiesta su gratitud al Programa de Magister en Ingeniería Industrial de la Universidad Católica de la Santísima Concepción por la Beca de Magíster otorgada.

\section{REFERENCIAS}

[1] F.I. Khan and A.K. Ghoshal. "Removal of volatile organic compounds from polluted air", J. Loss Prevent. Proc. Vol. 13, Issue 6, pp. 527-545. 2000.

[2] A.K. Ghoshal and S.D. Manjare. "Selection of appropriate adsorption technique for recovery of VOCs: an analysis". J. Loss Prevent. Proc. Vol. 15, Issue 6, pp. 413-421. 2002.

[3] H. Valdés, V.A. Solar, E.H. Cabrera, A.F. Veloso and C.A. Zaror. "Control of released volatile organic compounds from industrial facilities using natural and acid-treated mordenites: The role of acidic surface sites on the adsorption mechanism". Chem. Eng. J. Issue 244, pp. 117-127. 2014.

[4] R. Iranpour, H. Coxa, M. Deshusses and E. Schroeder. "Literature review of air pollution control biofilters and biotrickling filters for odor and volatile organic compound removal". Environ. Prog. Issue 24, pp. 254-267. 2005.

[5] S.H. Hsua, C.S. Huanga, T.W. Chunga and S. Gao. "Adsorption of chlorinated volatile organic compounds using activated carbon made from Jatropha curcas seeds". J. Taiwan Inst. Chem. E. Issue 45, pp. 2526-2530. 2014.

[6] United States Environmental Protection Agency (USEPA). "Taking toxics out of the air". Report EPA-452/K-00-002. 2000. Date of visit: 3 de enero del 2014. URL: http:// www.epa.gov

[7] J. Peñuelas y J. Lluisa. "Emisiones biogénicas de VOCs y cambio global". Ecosistemas. Redalyc. Vol. 12 N$^{\circ}$ 1, pp. 1-7. 2012.

[8] H. Sidebottom and J. Franklin. "The atmospheric fate and impact of hydrochlorofluorocarbons and chlorinated solvents". Pure Appl. Chem. Issue 68, pp. 1757-1769. 1996.

[9] K. van Craeynest, H. van Langenhove and R.M. Stuetz. "AOPs for VOCs and odour treatment". In: Simon Parsons (Ed.). Advanced Oxidation Processes for Water and Wastewater Treatment. Chapter 11. IWA Publishing. 2004.

[10] J. Lemus, M. Martin-Martínez, J. Palomar, L. Gómez-Sainero, M. Gilarranz and J. Rodriguez. "Removal of chlorinated organic volatile compounds by gas phase adsorption with activated carbón". Chem. Eng. J. Issue 211-212, pp. 246-254. 2012.

[11] M. Guillemot, J. Mijoin, S. Mignard and P. Magnoux. "Adsorption of perchloroethylene (PCE) in gas phase on zeolites of faujasite type: Influence of water vapour and of $\mathrm{Si} /$ Al ratio". Micropor. Mesopor. Mater. Issue 111, pp. 334-342. 2008.

[12] D. Mouvet, D. Barberis and A.C.M. Bourg. "Adsorption isotherms of tri- and perchloroethylene by various natural solids". J. Hydrol. Issue 149, pp. 163-1827. 1993.

[13] A.H. Englert and J. Rubio. "Characterization and environmental application of a Chilean natural zeolite". Int. J. Miner. Process. Issue 75, pp. 21-29. 2005.

[14] S.W. Baek, J.R. Kim and S.K. Ihm. "Design of dual functional adsorbent/catalyst system for the control of VOC's by using metal-loaded 
hydrophobic Y-zeolites". Catal. Today Issue 93-95, pp. 575-581. 2004.

[15] R. López-Fonseca, B. de Rivas, J.I. Gutiérrez-Ortiz, A. Aranzabal and J.R. González-Velasco. "Enhanced activity of zeolites by chemical dealumination for chlorinated VOC abatement". Appl. Catal. B-Environ. Issue 41, pp. 31-42. 2003.

[16] P.S. Chintawar and H.L. Greene. "Adsorption and catalytic destruction of trichloroethylene in hydrophobic zeolites". Appl. Catal. B-Environ. Vol. 14 Issue 1-2, pp. 37-47. 1997.

[17] B. Clausse, B. Garrot, C. Cornier, C. Paulin, M-H. Simonot-Grange and F. Boutros. "Adsorption of chlorinated volatile organic compounds on hydrophobic faujasite: correlation between the thermodynamic and kinetic properties and the prediction of air cleaning". Micropor. Mesopor. Mater. Issue 25, pp. 169-177. 1998.

[18] G. Pozan, Z. Ozcelik and I. Boz. "Total oxidation of toluene over metal oxides supported on a natural clinoptilolita-type zeolite". Chem. Eng. J. Issue 162, pp. 380387. 2010.

[19] A.Z. Abdullah, M.Z.A. Bakar and S. Bhatia. "Performance of H-ZSM-5-supported bimetallic catalysts for the combustion of polluting volatile organic compounds in air", J. Chem. Technol. Biot. Issue 80, pp. 10161025. 2005.

[20] M. Guillemot, J. Mijoin, S. Mignard and P. Magnoux. "Volatile organic compounds (VOCs) removal over dual functional adsorbent/catalyst system". Appl. Catal. B-Environ. Issue 75, pp. 249-255. 2007.

[21] G.S.P. Soylu, Z. Özçelik and I. Boz. "Total oxidation of toluene over metal oxides supported on a natural clinoptilolite-type zeolite". Chem. Eng. J. Vol. $162 \mathrm{~N}^{\mathrm{o}} 1$, pp. 380-387. 2010.

[22] Z. ÖzçelikG, S.P. Soylu and I. Boz. "Catalytic combustion of toluene over $\mathrm{Mn}, \mathrm{Fe}$ and $\mathrm{Co}-$ exchanged clinopozantilolite support". Chem. Eng. J. Vol. 155 Issue 1-2, pp. 94-100. 2009.

[23] L.Yosefi, M. Haghighi, S. Allahyari, R. Shokrani and S. Ashkriz. "Abatement of toluene from polluted air over $\mathrm{Mn} /$ Clinoptilolite-CeO2 nanopowder: Impregnation vs. ultrasound assisted synthesis with various Mn-loading". Adv. Powder Tech. In Press, Corrected Proof. 28 January, 2015.

[24] Z. Jamalzadeh, M. Haghighi and N. Asgari. "Synthesis and physicochemical characterizations of nanostructured $\mathrm{Pd} /$ carbon-clinoptilolite-CeO2 catalyst for abatement of xylene from waste gas streams at low temperature". Ind. Eng. Chem. J. Vol. $20 \mathrm{~N}^{\circ}$ 5, pp. 2735-2744. 2014.

[25] V. Kumar, A. Bhalla and A. Rathore. "Design of Experiments applications in bioprocessing: Concepts and approach". Biotechnol. Prog. AIChE. Vol. 30, Issue 1, pp. 86-99. 2014.

[26] Z. Zequan, Z. Haikui, L. Xin, S. Baochang, C. Jianfeng and L. Shao. "Ozonation of acidic phenol wastewater with $\mathrm{O} 3 / \mathrm{Fe}(\mathrm{II})$ in a rotating packed bed reactor: Optimization by response surface methodology". Chemical Engineering and Processing: Process Intesification. Vol. 60, pp. 1-8. 2012.

[27] B.D. Real, M.C. Ortiz and L.A. Sarabia. "Analysis of interferents by means a D-optimal screening design and calibration using partial least squares regression in the spectrophotometric determination of Cr VI)". Talanta. Vol. 71, Issue 4, pp. 1599-1609. 2007.

[28] C. Chih-Cherng. "Application of D-optimal design for modeling micro channel flow with different dispensing patterns". International Communications in Heat and Mass Transfer. Vol. 38, Issue 8, pp. 1061-1066. 2011.

[29] L. Eriksson, E. Johansson, N. Kettaneh-Wold, C. WikstrÄom and S. Wold. "Design of Experiments: Principles and Applications", UMETRIC Academy. Learnways AB, Umea, Sweden. ISBN: 91-97373-4-4. 2008.

[30] P. de Aguiar, F. Bourguignon, B. Khots, M.S. Massart and D.L. Phan-Than-Luu. "D-optimal Designs". Chemometrics and Intelligent Laboratory Systems. Vol. 30. Issue 2, pp. 199-210. 1995.

[31] M. Holena and M. Baerns. "Computer-Aided Design of Solid Catalysts. In: Handbook of Heterogeneous Catalysis". Wiley-VCH Verlag GmbH \& Co. KGaA, 2nd edn, pp. 66-81, Weinheim, Germany. ISBN-10: 3-527-31412. 2008.

[32] S. Corthals, T. Witvrouwen, P. Jacobs and B. Sels. "Development of dry reforming catalysts at elevated pressure: D-optimal 
vs. full factorial design". Catalysis Today, Vol. 159, Issue 1, pp. 12-24. 2011.

[33] S. Fang and R. Perera. "Damage identification by response surface based model updating using D-optimal design". Mechanical Systems and Signal Processing. Vol. 5, Issue 2, pp. 717-733. 2011.

[34] M. Muneer. "Optimization of process parameters using D-optimal design for Synthesis of $\mathrm{ZnO}$ nanoparticles via solgel technique". Journal of Industrial and Engineering Chemistry. Vol. 19, Issue 1, pp. 99-105. 2013.

[35] S. Alejandro, H. Valdes, M-H. Manero and C. Zaror. "BTX abatement using natural zeolite: the role of Bronsted acid sites". Water Sci. Technol. Vol. 66, Issue 8, pp. 1759-1765. 2012.

[36] H. Gutiérrez and S. de la Vara. "Análisis y diseño de experimentos". McGraw-Hill Interamericana. 2nd edn. pp. 338-446. México. ISBN-10: 970-10-6526-3. 2008.

[37] D. Montgomery. "Design and Analysis of Experiments". John Wiley and Sons. 5th edn., Arizona, USA. ISBN: 0-471-31649-0. 2001.
[38] D. Montgomery and R. Myers. "Response Surface Methodology: process and product optimization using designed experiment", John Wiley and Sons, 3nd ed., Canada. ISBN: 978-0-470-17446-3. 2002.

[39] Liu and Y-R Chiou. "Optimal decolorization efficien cy of Reactive Red 239 by UV/ $\mathrm{TiO} 2$ photocatalytic process coupled with response surface methodology". Chemical Engineering Journal. Vol. 112, Issue 1-3, pp. 173-179. 2005.

[40] A.N. Kolmogorov. "Sulla determinazione empirica di una legge di distribuzione". Giornale dell Ínstituto Italiano degli Attuari. Attuari. Vol. 4, pp. 83-91. 1933.

[41] N. Smirnov. "Sur les écarts de la courbe de distribution empirique". Recueli Mathematique. Vol. 6, Issue 1, pp. 3-26. 1939.

[42] H. Levene. "Robust tests for qguality of variance. In contributions to Probability and Statistics". Z. Olkin, ed. Stanford University Press, Palo Alto, CA., pp. 278292. 1960. 